\begin{tabular}{lll}
\hline VOL. 79 & 1999 & NO. 1 \\
\hline
\end{tabular}

\title{
THE BLOW-UP SOLUTIONS OF INTEGRAL EQUATIONS
}

BY

\section{WOJCIECH MYDLARCZYK (WROCŁAW)}

1. Introduction. We examine a class of Volterra equations motivated by models of processes in diffusive media which can have an explosive character. We consider

$$
u(t)=\int_{0}^{t} k(t-s) r(s) g(u(s)+h(s)) d s, \quad t>0,
$$

where

(i) $k(t)>0, K(t)=\int_{0}^{t} k(s) d s<\infty$ for $t>0$;

(ii) $r$ and $g$ are nondecreasing and continuous for $t \neq 0, r(t)=g(t)=0$ for $t \leq 0$, and $r(t), g(t)>0$ for $t>0$;

(iii) $h$ is nondecreasing, continuous, $h(t)=0$ for $t \leq 0$, and $h(t)>0$ or $h(t) \equiv 0$ for $t>0$.

Our considerations are motivated by the papers [7], [6], where such equations were investigated and some models of a diffusive medium which can experience explosive behaviour were provided.

One of such examples arises in the investigation of the shear bands formation in steel, which is accompanied by a dramatic rise in temperature when the material is subjected to very high strain rates. A theoretical model of that phenomenon has been developed recently in [2]. One particular variant of that model has the temperature $u(t)$ in the shear band governed by

$$
u(t)=\gamma \int_{0}^{t} \frac{(1+s)^{q}[u(s)+1]^{p}}{\sqrt{\pi(t-s)}} d s,
$$

where $\gamma, p, q$ are nonnegative material parameters related to the constitutive law for plasting straining.

We discuss blow-up solutions of (1.1) and give some criteria for their existence. In the case $k(s)=s^{\alpha-1}, \alpha>0$, important for applications, our results generalize those obtained in [6] and [7].

1991 Mathematics Subject Classification: Primary 45D05; Secondary 45G05, 45G10. Key words and phrases: nonlinear integral Volterra equations, blowing-up solutions. 
2. Statement of results. We are interested in continuous solutions $u \geq 0$ of (1.1). The existence of such solutions follows from the classical theorems (see [3]). Moreover, we prove that (1.1) has a maximal solution defined on its maximal interval of existence.

Theorem 2.1. Let $k, r, g$, h satisfy (i)-(iii). Then (1.1) has a maximal solution $u \geq 0$, which is a continuous nondecreasing function.

Concerning the uniqueness of solutions we have the following statement.

Theorem 2.2. Let $k, r, g, h$ satisfy (i)-(iii). If the maximal solution $u$ of (1.1) is positive for $t>0$, then it is a unique solution with that property.

From the physical point of view, only the solutions $u$ of (1.1) positive for $t>0$ are interesting. If $h(s)>0$ for $s>0$, then it is clear that the maximal solution $u$ of (1.1) must be positive for $t>0$. But if $h(s) \equiv 0$, we observe that $u \equiv 0$ is one of solutions to (1.1). In this case, the existence of solutions $u$ positive for $t>0$ requires additional assumptions on $g$ (see [4], [1]). We consider this problem in more detail for the equation

$$
u(t)=\int_{0}^{t}(t-s)^{\alpha-1} r(s) g(u(s)+h(s)) d s, \quad \alpha, t>0 .
$$

The following theorem gives the corresponding existence result.

Theorem 2.3. Let $r, g$ satisfy (ii) and $h \equiv 0$. Then the maximal solution $u$ of (2.1) is positive for $t>0$ if and only if

$$
\int_{0}^{\delta}\left[\frac{s}{g(s)}\right]^{1 / \alpha} \frac{d s}{s}<\infty
$$

Theorem 2.3 generalizes the results of [1], [4], where the case $r \equiv 1$ was considered and the analogous condition was obtained. We emphasize the fact that the existence of nontrivial solutions does not depend on the behaviour of $r$ near 0 .

We also analyse the problem of existence of blow-up solutions $u$ of $(2.1)$, that is, ones with $\lim _{t \rightarrow \widehat{t}-} u(t)=\infty, \widehat{t}<\infty$.

In the simple case $\alpha=1$, the equation (2.1) is an integral form of the initial value problem

$$
u^{\prime}(t)=r(t) g(u(t)+h(t)) \quad(t>0), \quad u(0)=0 .
$$

From the easily obtained relation

$$
\int_{s}^{t} \frac{u^{\prime}(\tau) d \tau}{g(u(\tau)+h(\tau))}=\int_{s}^{t} r(\tau) d \tau
$$


we infer that $u$ blows up if and only if

$$
\int_{\delta}^{\infty} \frac{1}{g(s)} d s<\infty
$$

In the general case we have the following statement.

Theorem 2.4. Let $r, g, h$ satisfy (ii). Then the maximal solution $u$ of (2.1) blows up if and only if

$$
\int_{\delta}^{\infty}\left[\frac{s}{g(s)}\right]^{1 / \alpha} \frac{d s}{s}<\infty, \quad \delta>0 .
$$

For $h \equiv 0$, we also give upper and lower bounds on the blow-up time $\widehat{t}$ of the maximal solution $u$ to (2.1) in implicit form:

Theorem 2.5. Let $r, g$ satisfy (ii) and $h \equiv 0$. Then the blow-up time $\widehat{t}$ of the maximal solution $u$ of (2.1) satisfies

$$
\alpha \int_{0}^{\widehat{t}} r(s)^{1 / \alpha} d s \leq \int_{0}^{\infty}\left[\frac{s}{g(s)}\right]^{1 / \alpha} \frac{d s}{s} \leq(\alpha+1) \widehat{\operatorname{tr}}(\widehat{t})^{1 / \alpha}
$$

if $0<\alpha \leq 1$, and

$$
\frac{\alpha\left(2^{1 / \alpha}-1\right)}{2^{1 / \alpha}} \int_{0}^{\hat{t} / 2} r(s)^{1 / \alpha} d s \leq \int_{0}^{\infty}\left[\frac{s}{g(s)}\right]^{1 / \alpha} \frac{d s}{s} \leq \alpha \int_{0}^{\widehat{t}} r(s)^{1 / \alpha} d s
$$

if $\alpha>1$.

Finally, we study the asymptotic growth of the maximal solution to (2.1) near the blow-up:

Theorem 2.6. Let $r, g$, h satisfy (i)-(iii) and $u$ be the maximal solution to (2.1) blowing up at $\widehat{t}$. Then there exist constants $c_{1}, c_{2}>0$ such that

$$
\frac{1}{3} I^{-1}\left(c_{1}(\widehat{t}-t)\right) \leq u(t) \leq I^{-1}\left(c_{2}(\widehat{t}-t)\right), \quad t \rightarrow \widehat{t}-,
$$

where $I^{-1}$ is the inverse function to

$$
I(\tau)=\int_{\tau}^{\infty}\left[\frac{s}{g(s)}\right]^{1 / \alpha} \frac{d s}{s}
$$

3. Auxiliary theorems. We begin with the comparison of a positive solution $u$ of (1.1) with a solution $v$ of the integral inequality

$$
v(t) \leq \int_{0}^{t} k(t-s) r(s) g(v(s)+h(s)) d s, \quad t>0 .
$$


THEOREM 3.1. Let $k, r, g, h$ satisfy (i)-(iii), $u$ be a solution of (1.1) positive for $0<t<\widehat{t}$ and $v$ be a continuous nonnegative solution of (3.1) for $0<t<\widehat{t}$. Then

$$
v(t) \leq u(t) \quad \text { for } t \in(0, \widehat{t}) .
$$

Proof. For any $c>0$ we define $v_{c}(t)$ as follows: $v_{c}(t)=0$ for $0<t \leq c$ and $v_{c}(t)=v(t-c)$ for $t \geq c$. Similarly, we define $h_{c}(t)$.

We easily check that

$$
v_{c}(t) \leq \int_{0}^{t} k(t-s) r(s) g\left(v_{c}(s)+h_{c}(s)\right) d s .
$$

Now we compare $u(t)$ and $v_{c}(t)$. Since $0=v_{c}(t)<u(t)$ for $0<t<c$, we notice that if $t_{0}$ is such that $v_{c}(t) \leq u(t)$ for $0<t<t_{0}$, then

$$
\begin{aligned}
u\left(t_{0}\right)-v_{c}\left(t_{0}\right) & \geq \int_{0}^{t_{0}} k(t-s) r(s)\left[g(u(s)+h(s))-g\left(v_{c}(s)+h_{c}(s)\right)\right] d s \\
& >\int_{0}^{c} k(t-s) r(s) g(u(s)+h(s)) d s>0 .
\end{aligned}
$$

Hence $v_{c}(t) \leq u(t)$ for $0<t<\widehat{t}$. Now letting $c \rightarrow 0$, we get our assertion.

Comparing any two solutions $u_{1}, u_{2}$ of (1.1) we get, by Theorem 3.1,

Corollary 3.1. Let $k, r, g$, h satisfy (i)-(iii). Then equation (1.1) has at most one continuous solution u positive for $t>0$.

Another corollary to Theorem 3.1 is the following.

Corollary 3.2. Let $k, r, g, h$ satisfy (i)-(iii). If $u$ is a continuous solution of (1.1) positive for $t>0$, then $u$ is nondecreasing.

Pr o of. We define

$$
\bar{u}(t)=\sup u(s) \quad \text { for } 0<s \leq t .
$$

Of course, $\bar{u}$ is continuous, nondecreasing and

$$
u(t) \leq \bar{u}(t), \quad \text { for } t>0,
$$

which gives

$$
u(t)=\int_{0}^{t} k(t-s) r(s) g(u(s)+h(s)) d s \leq \int_{0}^{t} k(t-s) r(s) g(\bar{u}(s)+h(s)) d s .
$$

Since the integral on the right-hand side is a nondecreasing function of $t$, which can be easily seen if one notices that $r(s) g(\bar{u}(s)+h(s))$ is a nondecreasing function and substitutes $\tau=t-s$ in the integral, the function $v(t)=\bar{u}(t)$ satisfies the inequality (3.1). Therefore, our assertion follows by Theorem 3.1 and (3.2). 
4. Proofs of theorems. Theorems 2.1 and 2.2 follow immediately from Corollaries 3.1 and 3.2.

Proof of Theorem 2.3. Necessity. Let $u$ be a nontrivial solution of (2.1). Then there exists $\delta>0$ such that

$$
u(t) \leq c \int_{0}^{t}(t-s)^{\alpha-1} g(u(s)) d s
$$

for $t \in[0, \delta]$ where $c=\sup _{s \in[0, \delta]} r(s)$. It is known (see [4], [1]) that the last inequality may hold for a nontrivial $u \geq 0$ if and only if the condition (2.2) is satisfied.

Sufficiency. First, we construct a nondecreasing subsolution $v$ of (2.1), that is, a nonnegative, nondecreasing function such that

$$
v(t) \leq T v(t)=\int_{0}^{t}(t-s)^{\alpha-1} r(s) g(v(s)) d s \quad \text { for } t \geq 0 .
$$

Such functions $v$ are equibounded, at least on a fixed small interval $[0, \delta]$, which follows from the easily obtained inequality

$$
\frac{v(t)}{g(v(t))} \leq \int_{0}^{t}(t-s)^{\alpha-1} r(s) d s .
$$

Let

$$
w(t)=\left(R^{-1} \circ \phi\right)(t) \quad \text { for } t>0,
$$

where

$$
R(t)=\int_{0}^{t} r(s)^{1 / \alpha} d s, \quad \phi(t)=\beta \int_{0}^{t}\left[\frac{s}{g(s)}\right]^{1 / \alpha} \frac{d s}{s}, \quad \beta=\frac{2^{2 / \alpha}}{\alpha\left(2^{1 / \alpha}-1\right)} .
$$

We easily check that

$$
w^{\prime}(s)=\beta \frac{1}{s}\left[\frac{s}{(r \circ w)(s) g(s)}\right]^{1 / \alpha}
$$

and hence

$$
w(t)=\beta \int_{0}^{t}\left[\frac{s}{(r \circ w)(s) g(s)}\right]^{1 / \alpha} \frac{d s}{s}, \quad t>0 .
$$

We define $v$ by its inverse

$$
v^{-1}(t)=w(t)+w\left(\frac{t}{2}\right) \quad \text { for } t>0 .
$$

We notice that

$$
v^{-1}(t)-v^{-1}\left(\frac{t}{2}\right) \geq w\left(\frac{t}{2}\right)-w\left(\frac{t}{4}\right) \geq\left[\frac{t}{(r \circ w)(t / 2) g(t / 2)}\right]^{1 / \alpha} .
$$


Now we get

$$
\int_{0}^{t}\left(v^{-1}(t)-v^{-1}(\tau)\right)^{\alpha} d m(\tau) \geq\left(v^{-1}(t)-v^{-1}\left(\frac{t}{2}\right)\right)^{\alpha} m\left(\frac{t}{2}\right) \geq t,
$$

where $m$ is the unique function such that $(m \circ v)(s)=r(s) g(v(s))$. The substitution $\tau=v(s)$ in the integral (4.2) shows that $v$ is a subsolution of (2.1).

Since $T^{n} v, n=1,2, \ldots$, is a nondecreasing bounded sequence of subsolutions of $(2.1)$, the desired solution $u$ can be obtained as the limit $u(t)=$ $\lim _{n \rightarrow \infty} T^{n} v(t), t \in(0, \delta)$, which ends the proof.

Consider the maximal nontrivial solution $u$ of (2.1). By Theorem 2.1, it is a nondecreasing function. Therefore integration by parts shows that

$$
u(t)=\frac{1}{\alpha} \int_{0}^{t}(t-s)^{\alpha} d(r(s) g(u(s)+h(s))) .
$$

It follows from (4.3) that $u$ is strictly increasing and we can introduce a nondecreasing function $m$ such that

$$
m(s)=\left(r \circ u^{-1}\right)(s) g\left(s+\left(h \circ u^{-1}\right)(s)\right), \quad s>0 .
$$

Now Theorem 1.1 of [5] gives

LEMmA 4.1. There exist constants $c_{1}, c_{2}>0$ such that

$$
c_{1}\left(u^{-1}\right)^{\prime}(t) \leq\left\{\int_{0}^{t}(t-s)^{\alpha-1} d m(s)\right\}^{-1 / \alpha} \leq c_{2}\left(u^{-1}\right)^{\prime}(t), \quad t>0 .
$$

Since the blow-up time $\widehat{t}$ of a nondecreasing solution $u$ is given by $\widehat{t}=$ $\lim _{t \rightarrow \infty} u^{-1}(t)$, a solution $u$ blows up if and only if

$$
\int_{A}^{\infty}\left\{\int_{0}^{t}(t-s)^{\alpha-1} d m(s)\right\}^{-1 / \alpha} d t<\infty
$$

for any $A>0$.

We are going to consider the integral in (4.5) in more detail. First, we get the following estimates.

Lemma 4.2. There exists a constant $c_{1}>0$ such that

$$
c_{1} I(2 A) \leq \int_{A}^{\infty}\left\{\int_{0}^{t}(t-s)^{\alpha-1} d m(s)\right\}^{-1 / \alpha} d t \leq I(A)
$$

if $0<\alpha \leq 1$, and

$$
I(2 A) \leq \int_{2 A}^{\infty}\left\{\int_{0}^{t}(t-s)^{\alpha-1} d m(s)\right\}^{-1 / \alpha} d t \leq 2 I(A), \quad A>0,
$$


if $\alpha \geq 1$, where

$$
I(A)=\int_{A}^{\infty}\left[\frac{s}{m(s)}\right]^{1 / \alpha} \frac{d s}{s} .
$$

Pr o of. For $\alpha \geq 1$, our assertion follows from the estimates

$$
2^{-\alpha+1} t^{\alpha-1} m(t / 2) \leq \int_{0}^{t}(t-s)^{\alpha-1} d m(s) \leq t^{\alpha-1} m(t), \quad t>0 .
$$

For $0<\alpha \leq 1$, the right inequality follows from the estimate

$$
\int_{0}^{t}(t-s)^{\alpha-1} d m(s) \geq t^{\alpha-1} m(t), \quad t>0 .
$$

We begin the proof of the left inequality with the estimate

$$
\begin{aligned}
\frac{1}{a} \int_{a}^{2 a}\left\{\int_{0}^{t}(t-s)^{\alpha-1} d m(s)\right\}^{-1 / \alpha} d t & \\
\geq & \left\{\frac{1}{a} \int_{a}^{2 a t} \int_{0}^{t}(t-s)^{\alpha-1} d m(s) d t\right\}^{-1 / \alpha} \quad(a>0)
\end{aligned}
$$

obtained by an application of the Jensen inequality. Combining it with the estimates

$$
\begin{aligned}
\int_{a}^{2 a} \int_{0}^{t}(t-s)^{\alpha-1} d m(s) d t & =\int_{0}^{a} \int_{a}^{2 a}(t-s)^{\alpha-1} d t d m(s)+\int_{a}^{2 a} \int_{s}^{2 a}(t-s)^{\alpha-1} d t d m(s) \\
& \leq \frac{1}{\alpha}(2 a)^{\alpha} m(2 a)
\end{aligned}
$$

and

$$
\int_{a}^{2 a}\left[\frac{s}{m(s)}\right]^{1 / \alpha} \frac{d s}{s} \leq \alpha\left(2^{1 / \alpha}-1\right) a^{1 / \alpha} m(a)^{-1 / \alpha}
$$

we obtain

$$
\text { (4.6) } \int_{a}^{2 a}\left\{\int_{0}^{t}(t-s)^{\alpha-1} d m(s)\right\}^{-1 / \alpha} d t \geq \frac{\alpha^{1 / \alpha-1}}{2\left(2^{1 / \alpha}-1\right) 2^{1 / \alpha}} \int_{2 a}^{4 a}\left[\frac{s}{m(s)}\right]^{1 / \alpha} \frac{d s}{s}
$$

for any $a>0$. Now, taking consecutively $a=2^{n} A, n=1,2, \ldots$, in (4.6) and summing up the resulting inequalities we get our assertion.

Now, we can pass to the proof of Theorem 2.4.

Proof of Theorem 2.4. By Lemma 4.2 and (4.5), the solution $u$ blows up if and only if

$$
\int_{A}^{\infty}\left[\frac{s}{r \circ u^{-1}(s) g\left(s+h \circ u^{-1}(s)\right)}\right]^{1 / \alpha} \frac{d s}{s}<\infty .
$$


If $u$ blows up, then $u^{-1}$ is bounded and (4.7) implies (2.3). Conversely, if (2.3) is satisfied, then (4.7) holds, so $u$ blows up. Thus the proof is complete.

Proof of Theorem 2.5. We obtain the results of Theorem 2.5 as a consequence of the following estimates for the maximal solution of the equation $(2.1)$.

LEMma 4.3. Let $r, g$ satisfy (ii) and $u$ be a nontrivial solution of (2.1). Then

$$
\begin{aligned}
\alpha^{(\alpha-1) / \alpha} \int_{0}^{t} r(s)^{1 / \alpha} d s & \leq \int_{0}^{u(t)}\left[\frac{s}{g\left(s+\left(h \circ u^{-1}\right)(s)\right)}\right]^{1 / \alpha} \frac{d s}{s} \\
& \leq(\alpha+1) \alpha^{-1 / \alpha} \operatorname{tr}(t)^{1 / \alpha}
\end{aligned}
$$

if $0<\alpha \leq 1$, and

$$
\begin{aligned}
\frac{\alpha\left(2^{1 / \alpha}-1\right)}{2^{2 / \alpha}} \int_{0}^{t / 2} r(s)^{1 / \alpha} d s & \leq \int_{0}^{u(t)}\left[\frac{s}{g\left(s+\left(h \circ u^{-1}\right)(s)\right)}\right]^{1 / \alpha} \frac{d s}{s} \\
& \leq \alpha^{(\alpha-1) / \alpha} \int_{0}^{t} r(s)^{1 / \alpha} d s
\end{aligned}
$$

if $\alpha>1$.

Proof. Let $m$ be defined by (4.4). Integrating by parts in (2.1) we get

$$
u(t)=\frac{1}{\alpha} \int_{0}^{t}(t-s)^{\alpha} d(m \circ u)(s) .
$$

If $0<\alpha \leq 1$, then an application of the Jensen inequality gives

$$
\frac{u^{\prime}(t)}{(m \circ u)(t)} \geq\left[\frac{\int_{0}^{t}(t-s)^{\alpha} d(m \circ u)(s)}{(m \circ u)(t)}\right]^{(\alpha-1) / \alpha} \text {. }
$$

Hence

$$
\frac{u^{\prime}(t)}{u(t)}\left[\frac{u(t)}{g(u(t)+h(t))}\right]^{1 / \alpha} \geq \alpha^{(\alpha-1) / \alpha} r(t)^{1 / \alpha} .
$$

For $\alpha \geq 1$ we obtain the opposite inequality in (4.11). Now, the left inequality in (4.8) and the right inequality in (4.9) follow from (4.11).

As in the proof of Theorem 2.2 in [4], we get

$$
\alpha^{1 / \alpha} \frac{u^{\prime}(t)}{u(t)}\left[\frac{u(t)}{(m \circ u)(t)}\right]^{1 / \alpha} \leq(\alpha+1)\left(1-L^{\prime}(t)\right),
$$


where $L(t)=\frac{1}{u(t)} \int_{0}^{t} u(s) d s \leq t$. Hence

$$
\alpha^{1 / \alpha} \frac{u^{\prime}(t)}{u(t)}\left[\frac{u(t)}{g(u(t)+h(t))}\right]^{1 / \alpha} \leq(\alpha+1)\left(1-L^{\prime}(t)\right) r(t)^{1 / \alpha} .
$$

Since $L(s) \leq s$ and $\int_{0}^{t}\left(1-L^{\prime}(s)\right) r(s)^{1 / \alpha} d s=(t-L(t)) r(t)^{1 / \alpha}-\int_{0}^{t}(s-L(s)) d\left(r(s)^{1 / \alpha}\right) \leq \operatorname{tr}(t)^{1 / \alpha}$ the right inequality in (4.8) follows immediately from (4.12).

Considering (4.10) as an integral equation with $m$ known and $u$ unknown, we construct a subsolution $v$ for it, in the same way as in the proof of Theorem 2.3. Then we have

$$
v(t) \leq u(t), \quad t>0 .
$$

Here $v$ is given by its inverse

$$
v^{-1}(t)=w(t)+w(t / 2),
$$

where

$$
\begin{gathered}
w(t)=\left(R^{-1} \circ \phi\right)(t), \quad R(t)=\int_{0}^{t} r(s)^{1 / \alpha} d s, \\
\phi(t)=\frac{2^{2 / \alpha}}{\alpha\left(2^{1 / \alpha}-1\right)} \int_{0}^{t}\left[\frac{s}{g\left(s+\left(h \circ u^{-1}\right)(s)\right)}\right]^{1 / \alpha} \frac{d s}{s} .
\end{gathered}
$$

Since $w(t)$ is nondecreasing, we get $u^{-1}(t) \leq 2 w(t)$. Now, an easy transformation gives $R(t / 2) \leq(\phi \circ u)(t)$, which is the left inequality in (4.9).

Now we consider the asymptotic behaviour of $u$ near the blow-up time.

Proof of Theorem 2.6. Suppose $u$ blows up. Then by Lemma 4.1 we have

$$
c_{1}(\widehat{t}-t) \leq \int_{u(t)}^{\infty}\left\{\int_{0}^{s}(s-\tau)^{\alpha-1} d m(\tau)\right\}^{-1 / \alpha} d s \leq c_{2}(\widehat{t}-t)
$$

where $c_{1}, c_{2}>0$ are some constants and $m$ is defined in (4.4). Hence by Lemma 4.2 we get

$$
c_{1}(\widehat{t}-t) \leq \int_{u(t)}^{\infty}\left[\frac{s}{\left(r \circ u^{-1}\right)(s) g\left(s+\left(h \circ u^{-1}\right)(s)\right)}\right]^{1 / \alpha} \frac{d s}{s}
$$

and

$$
\int_{2 u(t)}^{\infty}\left[\frac{s}{\left(r \circ u^{-1}\right)(s) g\left(s+\left(h \circ u^{-1}\right)(s)\right)}\right]^{1 / \alpha} \frac{d s}{s} \leq c_{2}(\widehat{t}-t) .
$$


Now, it suffices to note that for $t \rightarrow \widehat{t}-$ the integrals above can be estimated in terms of the integral $I$.

We illustrate the results of Theorem 2.6 by the following example.

EXAMPLE 4.1. Let $c_{1} z^{m}(\ln z)^{n} \leq g(z) \leq c_{2} z^{m}(\ln z)^{n}$ as $z \rightarrow \infty$, where $m>1, n \in \mathbb{R}$ and $c_{1}, c_{2}>0$ are some constants. Standard calculations show that $I^{-1}$ can be estimated as follows:

$$
c_{1} z^{\alpha /(1-m)}(-\ln z)^{n /(1-m)} \leq I^{-1}(z) \leq c_{2} z^{\alpha /(1-m)}(-\ln z)^{n /(1-m)}
$$

as $z \rightarrow 0+$, where $c_{1}, c_{2}>0$ are some constants. Now, by Theorem 2.6 we estimate the growth of the maximal solution $u$ to (2.1) near the blow-up time as follows:

$$
\begin{aligned}
& c_{1}(\widehat{t}-t)^{\alpha /(1-m)}(-\ln (\widehat{t}-t))^{n /(1-m)} \\
& \leq u(t) \leq c_{2}(\widehat{t}-t)^{\alpha /(1-m)}(-\ln (\widehat{t}-t))^{n /(1-m)}
\end{aligned}
$$

for $t \rightarrow \widehat{t}-$, where $c_{1}, c_{2}>0$ are some constants.

\section{REFERENCES}

[1] P. J. Bushell and W. Okrasiński, Uniqueness for a class of non-linear Volterra integral equations with convolution kernel, Math. Proc. Cambridge Philos. Soc. 106 (1989), 547-552.

[2] J. A. Dilellio and W. E. Olmstead, Shear band formation due to thermal flux inhomogeneity, SIAM J. Appl. Math. 57 (1997), 959-971.

[3] G. Gripenberg, S. O. Londen and O. Staffans, Volterra Integral and Functional Equations, Cambridge Univ. Press, 1990.

[4] W. Mydlarczyk, The existence of nontrivial solutions of Volterra equations, Math. Scand. 68 (1991), 83-88.

[5] - A condition for finite blow-up time for a Volterra equation, J. Math. Anal. Appl. 181 (1994), 248-253.

[6] W. E. Olmstead, C. A. Roberts and K. Deng, Coupled Volterra equations with blow-up solutions, J. Integral Equations Appl. 7 (1995), 499-516.

[7] C. A. Roberts, D. G. Lasseigne and W. E. Olmstead, Volterra equations which model explosion in a diffusive medium, ibid. 5 (1993), 531-546.

Mathematical Institute

University of Wrocław

Pl. Grunwaldzki 2/4

50-384 Wrocław, Poland

E-mail: mydlar@math.uni.wroc.pl 